\title{
XFEM Modeling of Cohesive Crack Propagation in Saturated Porous Media
}

\author{
E. Mikaeili ${ }^{1}$, M.T. Kazemi ${ }^{2}$, and S. Mohammadi ${ }^{3}$
}

\begin{abstract}
In this paper, the cohesive crack growth analysis has been investigated in saturated porous media. The XFEM algorithm is used for enrichment of displacement field, in which exploits the Heaviside step function as the enrichment function for representing the crack surfaces. The mass balance equation and the momentum balance of solid and fluid phases are used to reach the fully coupled set of equations in the framework of u-p formulation. The crack surfaces are assumed to be impermeable to ease the general procedure for the analysis of the cohesive crack growing. The nonlinearity imposed to the problem by accounting cohesive forces makes it necessary to implement Newton-Raphson iterative procedure to solve the nonlinear system of equations.
\end{abstract}

Index Terms-X-FEM method, Cohesive crack, Saturated porous media, Crack propagation, u-p formulation

\section{INTRODUCTION}

Introduction of extended finite element method by Belytschko and Black [1] and Moes et al.[2], facilitates the modeling of propagation of discontinuities that requires re-meshing in finite element method and proved to be a reliable and an efficient method for modeling strong discontinuities [2-4] and weak discontinuities [5,6].

Materials like soil and concrete physically possess huge amount of void spaces, in a way that affects the material behavior mechanically. The mechanics of porous materials are originated basically from the researches held by Terzaghi [7] and Biot [8], that since then has been the focus of so many number of studies.

Porous materials may undergo discontinuities by experiencing high external loadings. These discontinuities happened in the media may lead our system to initiating and propagation of cracks and ultimately failure. As a result, behavior of body including any discontinuities like cracks or shear bands can be extensively changed. In literature both singular and cohesive zone assumptions for modeling the tip of crack have been carried out to govern the mechanical behavior of the defects in porous media [9].

The behavior of the fracturing material, around the crack tip is nonlinear. In order to model this nonlinearity, the cohesive model is used. In this model, we have two main assumptions.

\footnotetext{
${ }^{1,2}$ Department of Civil Engineering, Sharif university of Technology,

${ }^{3}$ Department of Civil Engineering, University of Tehran,

Mikaeili_ehsan@mehr.sharif.ir, Kazemi@sharif.edu, smoham@ut.ac.ir
}

First, the near tip fracture processes are considered to occur through the crack line, in which in linear elastic fracture mechanics it is supposed to be occur at crack tip. Basic cohesive zone models have been proposed by [10], [11], in which in cohesive zone, a traction separation law relating the cohesive tractions to relative displacements is exploited.

In present paper, the extended finite element method is used for modeling cohesive crack propagation in saturated porous media. In order to account the crack opening, some modifications have been implemented to our formulation. In this regard, the displacement jump is captured through the model by adding enrichment functions across the fracture to the displacement field. However, the pressure field has been assumed to be continuous across the fracture.

\section{FORMULATION}

In this section, the governing equations for saturated porous media and the equations related to modeling the cohesive crack is presented. The momentum balance equation can be written as follows:

$$
\nabla . \sigma+\rho_{t} B=0
$$

Where $B$ is the body force and $\rho_{t}$ is the total density of the media, and can be formulated as,

$$
\rho_{t}=(1-n) \rho_{s}+n \rho_{f}
$$

Where $\rho_{s}$ and $\rho_{f}$ are the density of solid and fluid phase and $\mathrm{n}$ denotes the porosity. The total stress vector can be written in terms of the effective stress vector,

$$
\sigma=\sigma^{\prime}-\alpha m\left(\mathrm{p}_{f}\right)
$$

and the constitutive relation is defined in terms of the effective stress and strain increments,

$$
d \sigma^{\prime}=C(d \varepsilon)
$$

where $C$ is the elastic stiffness tensor and $\alpha$ is the Biot's constant,

$$
\alpha=1-\frac{K_{T}}{K_{S}}
$$

$$
\text { with } K_{T} \text { and } K_{S} \text { defined as the bulk modulus of porous }
$$

skeleton and solid grains, respectively. The momentum balance equation for the water phase leads to the generalized form of the Darcy equation,

$$
v^{d}=\frac{K}{\mu_{f v}}\left[-\nabla \mathrm{p}_{f}+\rho_{f} B\right]
$$


and the final form of the continuity equation for the water phase can be expressed as

$\left[\frac{(\alpha-n)}{K_{S}}+\frac{n}{K_{f}}\right] \frac{\partial \mathrm{p}_{f}}{\partial t}+\alpha \nabla \cdot v_{s}+\nabla \cdot\left[K\left(-\nabla \mathrm{p}_{f}+\rho_{f}(B)\right)\right]=0$

where $K$ is the permeability tensor $K_{S}$ and $K_{f}$ are defined as the bulk modulus and the Darcy velocity for the water phase, respectively. The boundary conditions include the imposed tractions and water fluxes on the outward boundaries and the corresponding prescribed essential boundary conditions for solid displacements and water pressures,

$$
\begin{aligned}
& u=\tilde{u} \\
& \mathrm{p}_{f}=\tilde{\mathrm{p}}_{f} \\
& \sigma . n_{\Gamma_{c}}=\bar{T} \\
& v_{f} \cdot n_{\Gamma_{c}}=\bar{Q}_{f}
\end{aligned}
$$

Additional condition is satisfied at fracture surfaces to account for compatibility of transformed cohesive tractions with the cohesive zone model adopted for the governing mechanical behavior of the fracture. In order to model the hydro-mechanical behavior of the fracture, the following internal boundary condition is applied,

$$
\sigma \cdot n_{\Gamma_{c}}=\bar{T}_{c}=\bar{T}_{d}-\alpha\left(\mathrm{p}_{f}\right) n_{\Gamma_{c}}
$$

where $n_{\Gamma_{c}}$ is the unit normal vector to the crack surface, $\bar{T}_{c}$ is the cohesive traction resulted from the cohesive law and $\mathrm{p}_{f}$ is the value of water pressure imposed on the crack surface. By applying the weighted residual method to equations, (1) and (7), with their corresponding boundary conditions, equations (9) and (10). The final weak form of equations will be obtained.

$$
\begin{gathered}
\int_{\Omega} \delta\left(\mathrm{L}_{u} u\right)^{T}(\sigma) d \Omega-\int_{\Omega} \delta u^{T} B d \Omega \\
-\int_{\Gamma_{t}} \delta u^{T} T d \Gamma_{t}+\int_{\Gamma_{c}} \delta u^{T} \bar{T}_{c} d \Gamma_{c}=0 \\
\int_{\Omega} \delta\left(\mathrm{p}_{f}\right)^{T} \cdot\left[\frac{(\alpha-n)}{K_{S}}+\frac{n}{K_{f}}\right] \frac{\partial \mathrm{p}_{f}}{\partial t} d \Omega-\int_{\Omega} \delta\left(\mathrm{p}_{f}\right)^{T} \alpha m^{T} L_{u} \frac{\partial u}{\partial t} d \Omega \\
-\int_{\Omega} \delta\left(\mathrm{L}_{p f} \mathrm{p}_{f}\right)^{T} \cdot\left[K\left(-\nabla \mathrm{p}_{f}+\rho_{f}(B)\right)\right] \mathrm{d} \Omega=0
\end{gathered}
$$

$\delta u$ and $\delta \mathrm{p}_{f}$ represent the variations of solid displacement and water pressure, respectively and act as the weighting functions in the proposed Galerkin weak form of the fully coupled saturated porous medium. The integrals on the crack surfaces $\left(\Gamma_{c}\right)$ are the interfacial terms resulted from the hydro-mechanical exchange of tractions through the fracture walls. The differential operators $D_{u}$ and $D_{p}$ are defined as,

$$
D_{u}=\left[\begin{array}{cc}
\frac{\partial}{\partial x} & 0 \\
0 & \frac{\partial}{\partial y} \\
\frac{\partial}{\partial y} & \frac{\partial}{\partial x}
\end{array}\right], D_{p}=\left[\begin{array}{c}
\frac{\partial}{\partial x} \\
\frac{\partial}{\partial y}
\end{array}\right]
$$

\section{NUMERICAL DISCRETIZATION AND NON-LINEAR SOLUTION}

In order to model the displacement and pressure field, the following enriched local variables are defined;

$$
\begin{aligned}
& u(x)=\sum_{i} N_{i}(x) u_{i}+\sum_{j} N_{j}(x) H(x) a_{j} \\
& p_{f}(x)=\sum_{i} N_{i}^{p}(x) p_{i}
\end{aligned}
$$

where $N_{i}(x)$ and $N_{i}^{p}(x)$ stand for the standard finite element shape functions for discretization of the solid displacements and water pressures, respectively. $u_{i}$ and $a_{j}$ are the standard and enrichment degrees of freedom. $\mathrm{H}(\mathrm{x})$ is the Heaviside enrichment function (15) representing the discontinuous part of the displacement field.

$$
H(x)= \begin{cases}1 & \text { if }\left(x-x^{c r}\right) \cdot \mathrm{n} \geq 0 \\ 0 & \text { otherwise }\end{cases}
$$

Insertion of the enriched approximation (14) into the weak form equations of (11) and (12) results in the complete discretized set of constrained equations for the fully coupled saturated deforming porous medium,

$\int_{\Omega} B^{T, n+1} \sigma^{\prime} d \Omega-Q_{s f}^{n+1} \mathrm{p}_{f}^{n+1}+f_{c}^{n+1}=F_{u}^{n+1}$

$Q_{f s}^{n+1} \dot{U}^{n+1}+p_{f f}^{n+1} \dot{p}_{f}^{n+1}+H_{f f}^{n+1} p_{f}^{n+1}=F_{f}^{n+1}$

along with the following internal force,

$$
f_{c}=\int_{\Gamma_{c}} \llbracket N_{u}^{T} \rrbracket \bar{\Gamma}_{c} \cdot d \Gamma_{c}
$$

Where,

$$
\begin{aligned}
& K=\int_{\Omega} B_{u}^{T} C B_{u} d \Omega \\
& Q_{s f}=\int_{\Omega} B_{u}^{T} \alpha m N_{p f} d \Omega \\
& Q_{f s}=\int_{\Omega} N_{p f}^{T} \alpha m^{T} \cdot B_{u} d \Omega \\
& P_{f f}=\int_{\Omega} N_{p f}^{T} \cdot\left(\frac{1}{Q_{f f}}\right) \cdot N_{p f} d \Omega \\
& H_{f f}=\int_{\Omega}\left(L_{p f} N_{p f}\right)^{T} K\left(\mathrm{~L}_{p f} N_{p f}\right) \\
& N_{k}(x)=\delta\left(x-x_{k}\right)
\end{aligned}
$$

$N_{k}(x)$ is defined as the set of nodes placed along the essential boundaries and, 


$$
\begin{aligned}
& B_{u}=\left[\begin{array}{ll}
B_{u}^{s t d} B_{u}^{H v} &
\end{array}\right] \\
& N_{u}=\left[\begin{array}{ll}
N_{u}^{s t d} & N_{u}^{H v}
\end{array}\right] \\
& N_{p f}=\left[N_{p f}^{s t d}\right]
\end{aligned}
$$

and using the Newton-Raphson algorithm, the final form of the discretized set of equations can be written as,

$$
\begin{aligned}
& R_{u}^{i, n+1}=K U^{i, n+1}-Q_{s f} P_{f}^{i, n+1}+f_{c}^{n+1}-F_{u}^{n+1} \\
& R_{p f}^{i, n+1}=Q_{f s}^{n+1}\left(\frac{U^{n+1}-U^{n}}{\Delta t}\right)+P_{f f}^{n+1}\left(\frac{P_{f}^{n+1}-P_{f}^{n}}{\Delta t}\right) \\
& +H_{f f}^{n+1} P_{f}^{n+1}-F_{f}^{n+1}
\end{aligned}
$$

and the residual and unknown vectors can be expressed by:

$$
\begin{aligned}
& R^{i, n+1}=\left\{\begin{array}{l}
R_{u}^{i, n+1} \\
R_{p f}^{i, n+1}
\end{array}\right\} \\
& X^{i, n+1}=\left\{\begin{array}{l}
U^{i, n+1} \\
P_{f}^{i, n+1}
\end{array}\right\}
\end{aligned}
$$

Differentiating the residual with respect to the unknowns vector $\mathrm{X}$, gives the Jacobian matrix for the constrained system of equations,

$$
J=\left[\begin{array}{ll}
K+\frac{\partial f_{c}}{\partial u} & -Q_{s f}+\frac{\partial f_{c}}{\partial p_{f}} \\
\frac{Q_{f s}}{\Delta t} & \frac{P_{f f}}{\Delta t}+H_{f f}
\end{array}\right]
$$

The following fully coupled nonlinear system of equations should then be solved at each time step to compute the vector of unknown increments,

$$
R^{i+1, n+1}=R^{i, n+1}+J\left[d X^{i+1, n+1}\right]=0
$$

in which $R^{i, n+1}$ is given in (21),(22) and is computed as following:

$$
R^{i, n+1}=J\left[\begin{array}{l}
U^{i, n+1} \\
P_{f}^{i, n+1}
\end{array}\right]-\left[\begin{array}{c}
F_{u} \\
F_{f}
\end{array}\right]-P^{n}
$$

where $P^{n}$ is the value of converged solution from the last time step of analysis:

$$
P^{n}=\left[\begin{array}{c}
0 \\
Q_{f s}\left(\frac{U^{n}}{\Delta t}\right)+P_{f f}\left(\frac{P_{f}^{n}}{\Delta t}\right)
\end{array}\right]
$$

Then, the unknowns vector is updated at each iteration until the required convergence criterion is satisfied:

$$
\left[\begin{array}{l}
U^{i+1, n+1} \\
P_{f}^{i+1, n+1}
\end{array}\right]=\left[\begin{array}{l}
U^{i, n+1} \\
P_{f}^{i, n+1}
\end{array}\right]+\left[\begin{array}{l}
d U^{i+1, n+1} \\
d P_{f}^{i+1, n+1}
\end{array}\right]
$$

\section{NUMERICAL MODELING}

The problem is analyzed, assuming a fully saturated medium. The notched square plate has a length $50 \mathrm{~mm}$ and is located along the symmetry axis. The geometry and loading of the plate are shown in Fig.1. The plate is loaded in pure modeI by two vertical velocities of $2.35 \times 10^{-5}$.

All boundaries of the plate are assumed to be impermeable. The analysis is carried for a discretization of bilinear quadrilateral elements with time steps of $0.01 \mathrm{~s}$. The numerical analysis continues until the crack tip has reached the right hand side of the plate. Fig. 3 shows the contours of the pressure fields at time steps and Fig. 4, shows the contours of pressure gradient. At last time step, the stress fields across the domain is displayed in Fig. 5. Also material properties are presented in table.1.

TABLE I: MATERIAL PROPERTIES

\begin{tabular}{lc}
\hline Young's modulus $(\mathrm{Pa})$ & $25.85 \times 10^{9}$ \\
Solid bulk modulus $(\mathrm{Pa})$ & $13.46 \times 10^{9}$ \\
Fluid bulk modulus $(\mathrm{Pa})$ & $0.2 \times 10^{9}$ \\
Water viscosity (MPa s) & $1.0 \times 10^{-9}$ \\
Intrinsic permeability of water (m2) & $2.78 \times 10^{-9}$ \\
Poisson's ratio & 0.18 \\
Biot's coefficient & 1 \\
Tensile strength $(\mathrm{MPa})$ & 2.7 \\
Fracture energy $(\mathrm{N} / \mathrm{mm})$ & 0.095 \\
Porosity & 0.2 \\
\hline
\end{tabular}

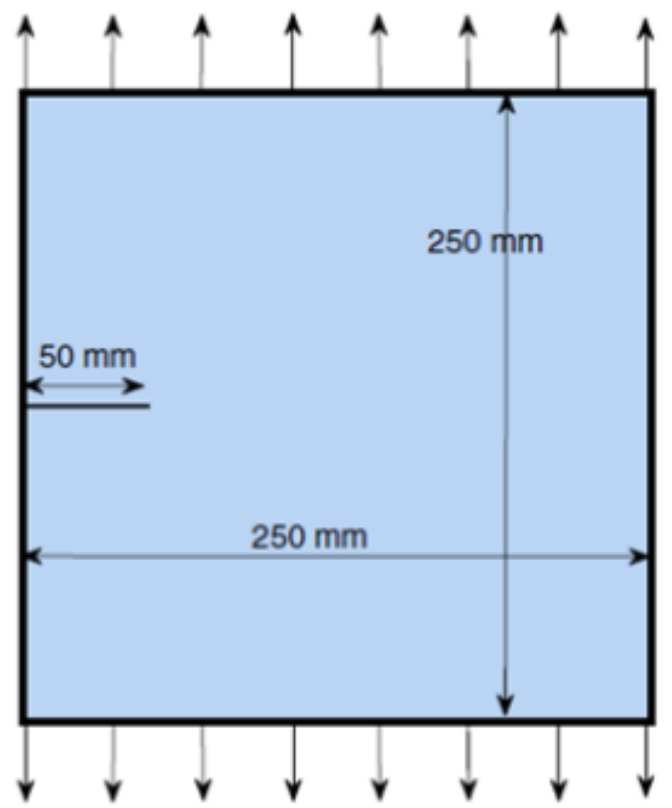

Step

Fig. 1. Propagation of a cohesive crack in a saturated porous medium 

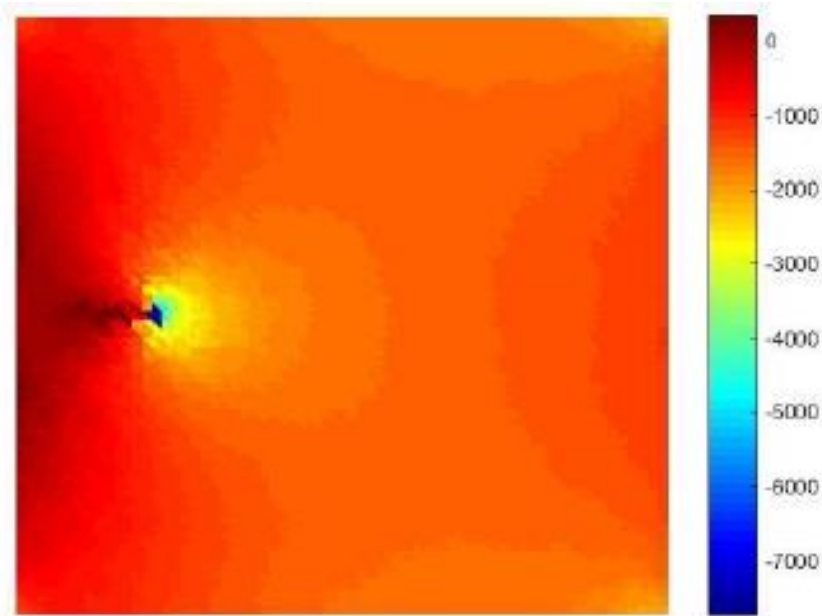

Step 1
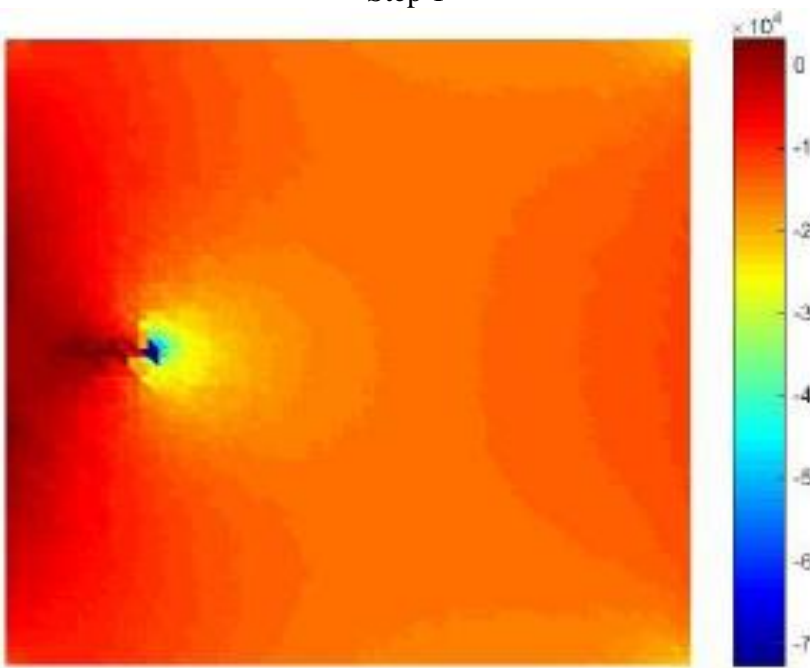

Step 100

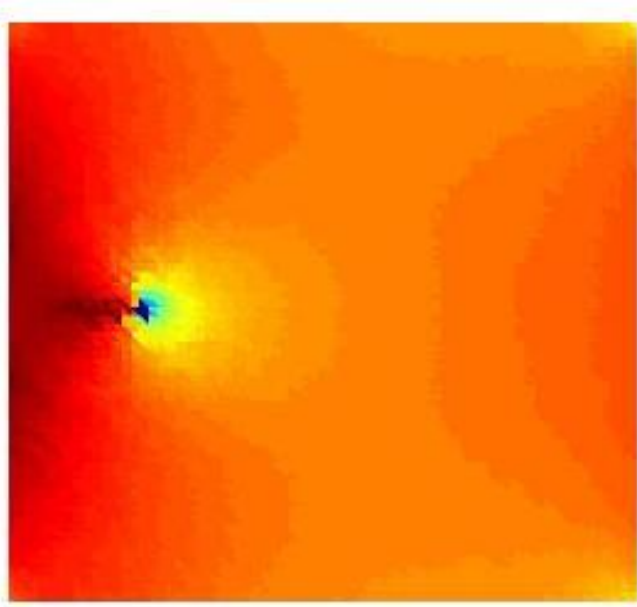

Step 200

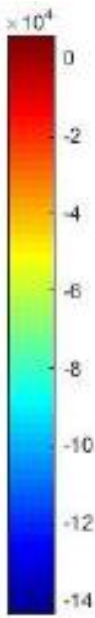

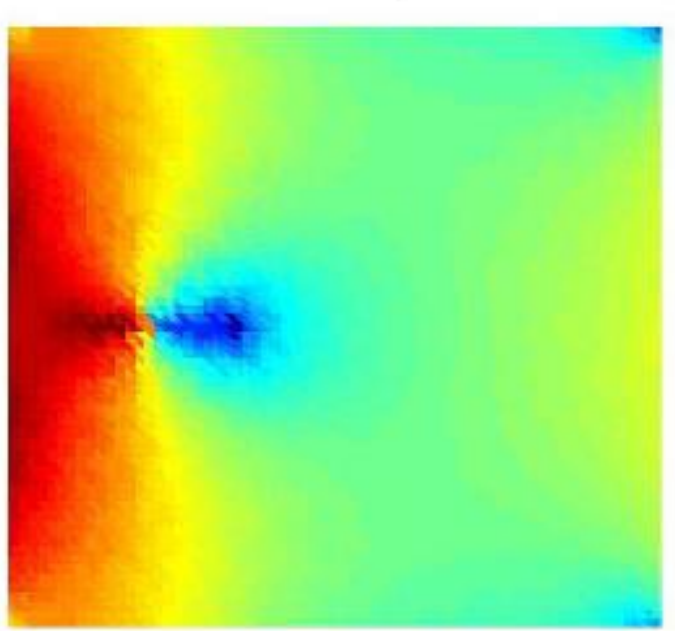

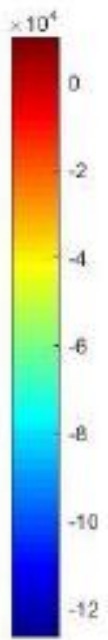

Step 400
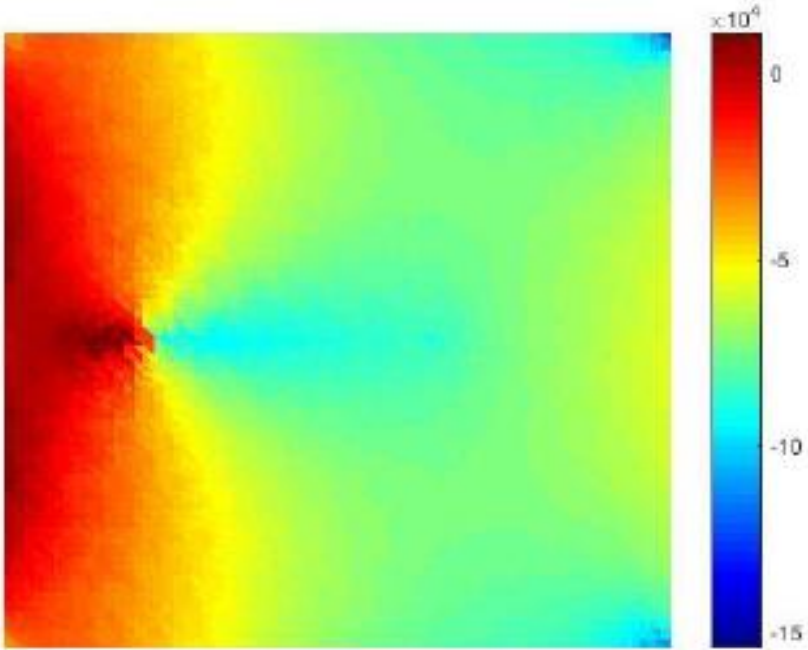

Step 500

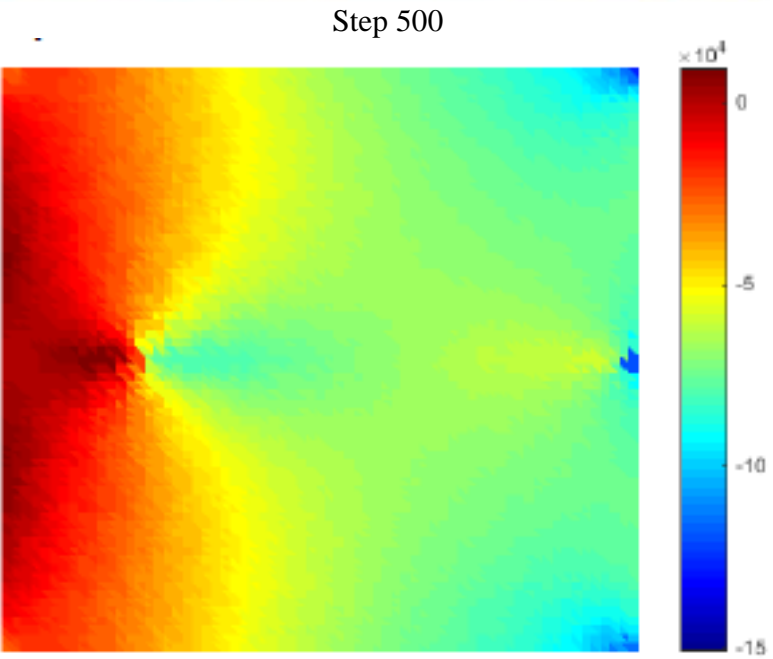

Step 600

Fig. 2: Pressure Field at different steps of time of the analysis for the mode I cohesive crack propagation in a saturated porous media with no coupling term (values in $\mathrm{Pa}$ ) 


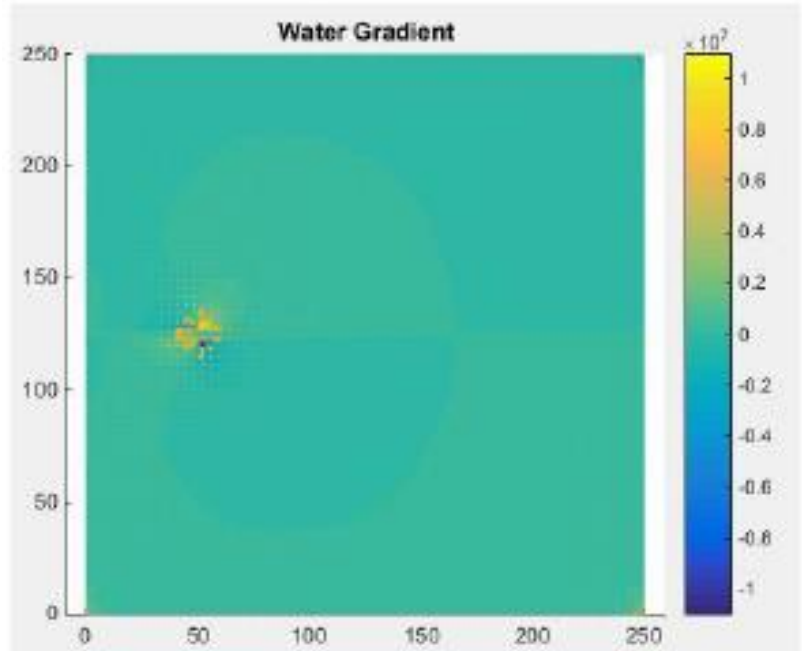

Step 1

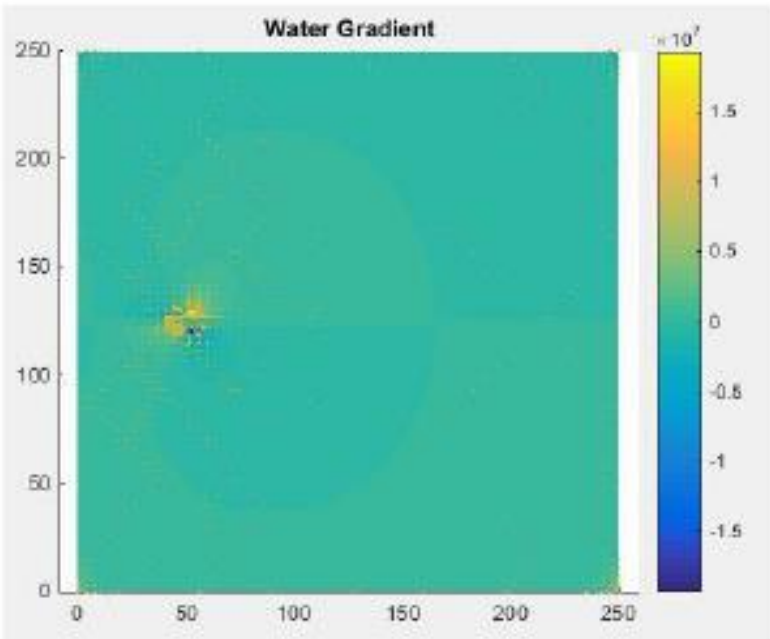

Step 200
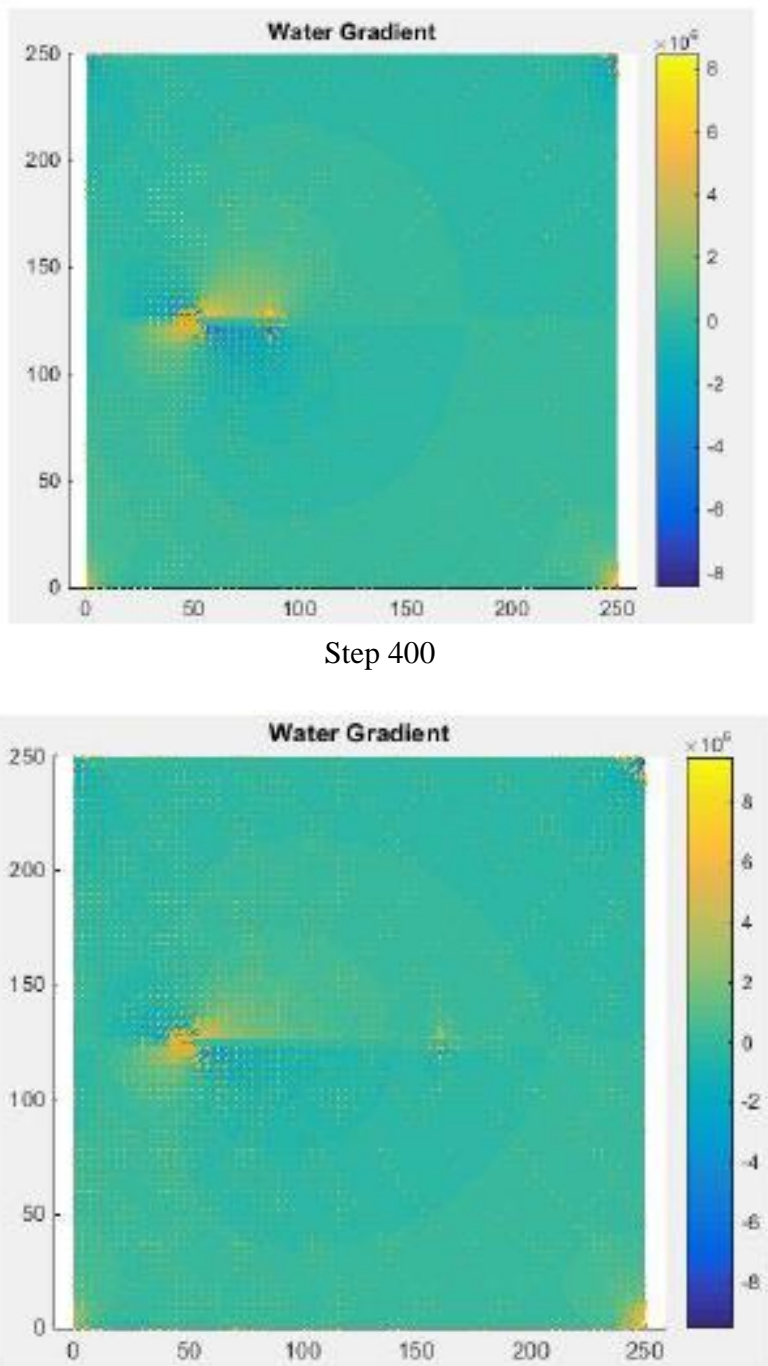

Step 500

Fig. 3: Water gradient at different steps of time
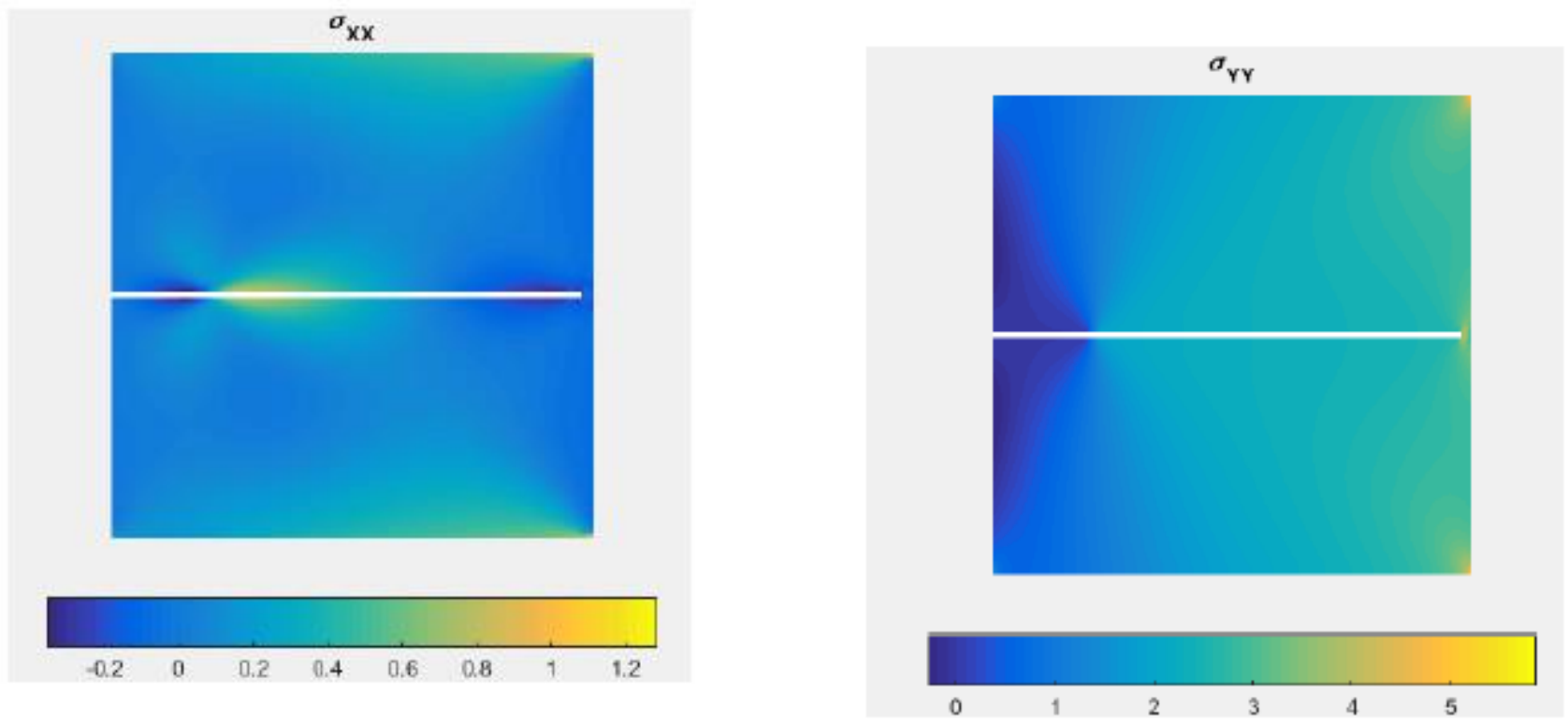

Fig. 4: Stress fields at last time step (values in Mpa) 


\section{CONCLUSION}

In this paper extended finite element method is utilized for modeling cohesive crack discontinuities in saturated porous media. This theory proved to be a sufficient method to capture the strong discontinuities. And helps to model the discontinuities with acceptable accuracy without necessity of resmeshing and implementing refined meshing.

\section{REFERENCES}

[1] T. Belytschko, T. Black, "Elastic crack growth in finite elements with minimal remeshing," vol. 63 (55), pp. 760-788, 1999.

[2] N. Moes, J. Dolbow, T. Belytschko, "A finite element method for crack growth without remeshing," International Journal of Numerical Methods in Engineering, vol. 46, pp.131-150, 1999.

[3] S. Mohammadi, Extended finite element method, Blackwell, 2007.

[4] S. Mohammadi, XFEM fracture analysis of composites, Wiley, 2012.

[5] J. V. Cox, "An extended finite element method with analytical enrichment for cohesive crack modeling," International Journal of Numerical Methods in Engineering. 78, pp. 48-83, 2009.

[6] P. Dumstorff, G. Meschke, "Crack propagation criteria in the framework of XFEM based structural analyses," International Journal of Numerical and Analytical Methods in Geomechanics. 31:239-59, 2007.

[7] K. Terzaghi, "Theoretical soil mechanics", Wiley, 1943.

[8] M. A. Biot,"General theory of three-dimensional consolidation", J Appl Phys. Vol. 12, pp. 155-64, 1941.

[9] J. Rethore, R. Borst, M. A. Abellan, "A two-scale model for fluid flow in an unsaturated porous medium with cohesive cracks" Computational Mechanics. 42:227-38, 2008.

[10] G. I. Barenblatt, "The formation of equilibrium cracks during brittle fracture: general ideas and hypotheses. Axially symmetric cracks", Journal of Applied Mathematics and Mechanics. 23:622 - 636, 1959.

[11] G. I. Barenblatt, "The Mathematical theory of equilibrium cracks in brittle fracture", Advances in Applied Mechanics. 7:55 - 129, 1962.

[12] T. Mohammadnejad, A. R. Khoei, "Hydro-mechanical modeling of cohesive crack propagation in multiphase porous media using the extended finite element method", International Journal of Numerical and Analytical Methods in Geomechanics.37. pp.47-79, 2013.

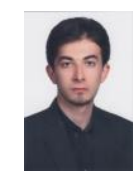

He is from Iran. His birthdate is 1991/september/1.

$\mathrm{He}$ is graduate student from Sharif University of Technology in department of civil engineering, Tehran, Iran. He earned his master in 2015.

Mr. Ehsan Mikaeili

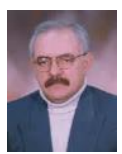

He Lives in Tehran.

He is Professor at Sharif University of Technology, in department of civil engineering. He was graduated from Northwestern university, department of civil engineering.

Prof. M.T. Kazemi

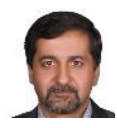

He lives in Tehran

$\mathrm{He}$ is professor at university of Tehran, in department of civil engineering. He was graduated from Swansea university, Prof. S. Mohammadi department of Computational Mechanics. 